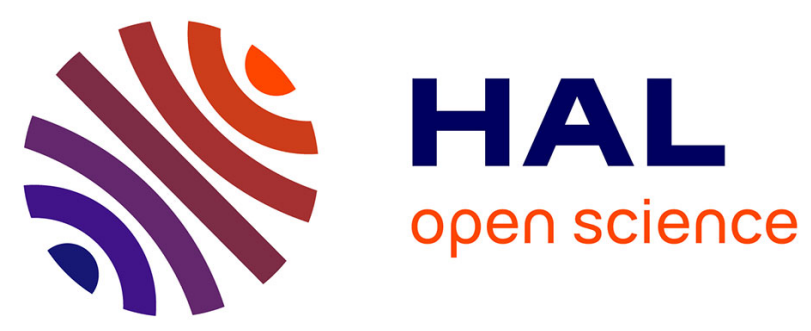

\title{
Mining Event Logs to Assist the Development of Executable Process Variants
}

Ngoc Chan Nguyen, Karn Yongsiriwit, Walid Gaaloul, Jan Mendling

\section{To cite this version:}

Ngoc Chan Nguyen, Karn Yongsiriwit, Walid Gaaloul, Jan Mendling. Mining Event Logs to Assist the Development of Executable Process Variants. 26th International Conference on Advanced Information Systems Engineering, Jun 2014, Thessaloniki, Greece. pp.548 - 563, 10.1007/978-3-319-07881-6_37 . hal-01113532

\section{HAL Id: hal-01113532 \\ https://hal.inria.fr/hal-01113532}

Submitted on 5 Feb 2015

HAL is a multi-disciplinary open access archive for the deposit and dissemination of scientific research documents, whether they are published or not. The documents may come from teaching and research institutions in France or abroad, or from public or private research centers.
L'archive ouverte pluridisciplinaire HAL, est destinée au dépôt et à la diffusion de documents scientifiques de niveau recherche, publiés ou non, émanant des établissements d'enseignement et de recherche français ou étrangers, des laboratoires publics ou privés. 


\title{
Mining Event Logs to Assist the Development of Executable Process Variants
}

\author{
Nguyen Ngoc Chan ${ }^{1}$, Karn Yongsiriwit ${ }^{2}$, Walid Gaaloul ${ }^{2}$, and \\ Jan Mendling 3 \\ 1 Université de Lorraine, Loria UMR 7503, France \\ 2 Telecom SudParis, Samovar UMR 5157, France \\ 3 Institute for Information Business \\ Wirtschaftsuniversität Wien (WU Vienna), Austria
}

\begin{abstract}
Developing process variants has been proven as a principle task to flexibly adapt a business process model to different markets. Contemporary research on variant development has focused on conceptual process models. However, process models do not always exist, even when process logs are available in information systems. Moreover, process logs are often more detailed than process models and reflect more closely to the behavior of the process. In this paper, we propose an activity recommendation approach that takes into account process logs for assisting the development of executable process variants. To this end, we define a notion of neighborhood context for each activity based on logs, which captures order constraints between activities with their occurrence frequency. The similarity of the neighborhood context between activities provides us then with a basis to recommend activities during the process of creating a new process model. The approach has been implemented as a plug-in for ProM. Furthermore, we conducted experiments on a large collection of process logs. The results indicate that our approach is feasible and applicable in real use cases.
\end{abstract}

Keywords: process mining · business process design · neighborhood context $\cdot$ context matching

\section{Introduction}

A process variant is an adjustment of a business process to flexibly adapt the business model to a specific context. Enterprises or organizations usually need to support many variants of the same process due to constraints from regulations, geography, religion, etc. For example, car rental companies, such as Hertz, Avis or Sixt, need to customize their reservation process to follows laws in a country or culture of a region. Suncorp, one of the largest Australian insurance group, has developed more than 30 different variants of the process of handling an insurance claim [32].

In recent years, there have been many efforts on facilitating the development of business process variants such as (i) using available reference models to be individualized to fit the requirements $[31,14]$, or (ii) finding existing similar 
models to inspire the new process design $[35,4,15]$. However, the design with reference models is still labor-intensive, which is often the cause for error-prone and time-consuming design [3]. Meanwhile, recommending entire process models costs much computation time, especially when the number of activities is large. Large models are also not handy for a designer who needs to pick a specific piece of functionality from them. In this context, it is desirable to recommend only a small but well-selected set of activities in order to help the designer.

Prior research has emphasized the advantages of recommendations during process model design [21,11-13]. However, business process models do not always exist in large-scale information systems such as ERP, CRM, or workflow management systems [1], even when process logs are available. Executable process models also require detailed technical activities to be recommended, while conceptual process models often describe processes in a coarse-granular way [6]. Moreover, process models do not explicitly show the importance of activities or connection flows, which can be a valuable parameter to compute more precisely the similarity between two activities. Meanwhile, this information is recorded in process event logs in the form of traces and their frequency.

In this paper, we propose an approach that builds upon process event logs for making activity recommendations during the development of executable process variants. We examine the relation between activities based on their execution order and frequency as recorded in logs. We define the notion of a neighborhood context of an activity as a fragment of the log-based model that contains the considered activity and relations to its neighbors. Relations between activities and their occurrence frequency provide the basis for the computation of the similarity between activities. In the process design phase, this similarity is used to recommend activities which might be closely related to the one that has just been added to the model.

The rest of the paper is organized as follows. Section 2 presents an example that motivates our approach. Section 3 continues with the process model and the neighborhood context graph extracted from logs. Section 4 defines the recommendation approach. Section 5 presents the implementation and experimental results. Section 6 discusses related work before Section 7 concludes the paper.

\section{Motivating example}

Consider a process designer who is about to develop a new variant of a liability claim process of an insurance company (inspired from[28]). She starts her work by either retrieving an existing process or quickly designing a new one based on her experiences (Fig. 1): first, some data related to the claim is registered (cf. activity A), and then a full check is performed (B). Afterwards, the claim will be evaluated (D), and then it is either rejected (F) or approved (E and G). Finally, the case is archived and closed $(\mathrm{H})$.

To develop a new variant, the designer may need recommendations for certain positions of the current process. Our approach assist her by providing recommended activities for at each selected position. For example, in Fig. 2, $\mathrm{K}$ is recommended for $\mathrm{A}, \mathrm{C}$ is recommended for $\mathrm{B}$ and so on. These recommenda- 
tions possibly help her easily have new ideas to design a new process variant, for instance, as given in Fig. 3 .

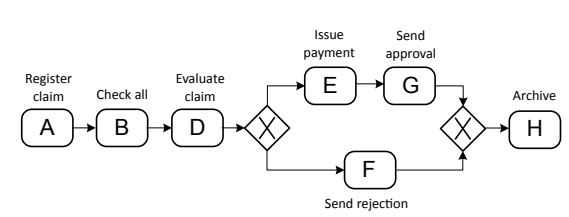

Fig. 1: Initial design

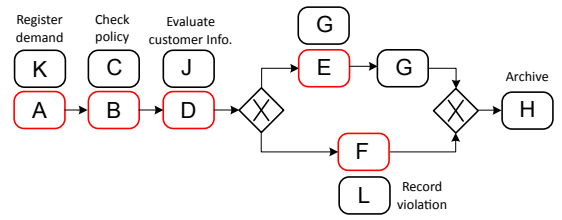

Fig. 2: Activity recommendation

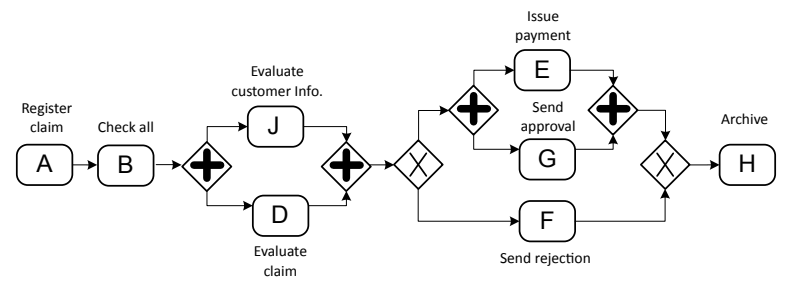

Fig. 3: A new variant

\begin{tabular}{|c|c|c|}
\hline Traces & Log traces & Repeat \\
\hline 1 & ACDGEH & \\
2 & ABDFH & \\
3 & ABDEGH & \\
4 & ABDFH & trace 2 \\
5 & ACDGEH & trace 1 \\
6 & ABDGEH & \\
7 & ACDFH & \\
\hline
\end{tabular}

Table 1: Event logs of the liability claim process $([28])$

\begin{tabular}{|c|c|c|}
\hline Traces & Log traces & Repeat \\
\hline 1 & KBJFH & \\
2 & KBJGH & \\
3 & KBJFH & trace 1 \\
4 & KBJFH & trace 1 \\
5 & KBJGH & trace 2 \\
\hline
\end{tabular}

Table 2: Event $\operatorname{logs}$ of a customer subscription process

Recommendations for each activity are made based on their similarity. The computation of this similarity can be based on process event logs even when a a-priori process model is not available.

To elaborate the steps to recommendations in our approach, we use the example of a liability claim process within an insurance company that was presented in [28] and another example of a customer subscription process. Table 1 and Table 2 present the process event logs of these processes. Each process instance is recorded as a trace. Each trace is a sequence of activities, which presents their execution order within a process instance. In a trace, the following activity (activity on the right) is performed after the followed activity (activity on the left). For example, consider a trace $\sigma=A B C D$, activity $\mathrm{B}$ is performed after activity $\mathrm{A}$ and before activity $\mathrm{C}$. The third column of Table 1 and Table 2 presents the repetition of a trace that was previously executed. 
In the following, we present an approach to build log-based business process models based on process event logs. These models provide the basis for defining a notion of activity neighborhood context, which we utilize for computing the similarity between activities.

\section{Exploiting activity neighborhood context from logs}

In this section, we present the log-based business process and the neighborhood context that are captured from business process logs. We firstly present some definitions related to business process logs (section 3.1). Then, we present definitions of the log-based business process (section 3.2 ) and the activity neighborhood context (section 3.3).

\subsection{Preliminaries}

According to [1] and [29], a business process log is defined as follows.

Definition 1 (Log trace, business process $\log , L)$. Let $A$ be a set of activities. $A^{*}$ denotes the set of finite sequences over $A$ and $\sigma=a_{1} a_{2} \ldots a_{n} \in A^{*}$ is a $\log$ trace. $L \in \mathcal{P}\left(A^{*}\right)$ is a business process $\log ^{4}$.

As explained in $[1,29]$, a business process log does not consider the repetition of a trace. For example, in Table $1, L$ includes only traces $1,2,3,6$ and 7 . Traces 4 and 5 are excluded by $L$ as they repeat traces 2 and 1 . In our approach, we extend Definition 1 to define the full business process log (see Definition 2) that includes all $\log$ traces.

Definition 2 (Full business process log, $L^{*}$ ). A full business process log is the business process log that includes all executed traces. The full business log is denoted by $L^{*}, L^{*} \in \mathcal{P}^{*}\left(A^{*}\right) . L \subseteq L^{*}$.

For example, in Table $1, L^{*}$ includes all traces from 1 to 7 . In Table $2, L^{*}$ includes all traces from 1 to 5 , while $L$ includes only trace 1 and 2 .

Definition 3 (Log-based ordering relation, $>_{L}$ ). Let $L$ be a business process $\log$ over $A$, i.e., $L \in \mathcal{P}\left(A^{*}\right)$. Let $a, b \in A$. $a>_{L} b$ iff $\exists \sigma=a_{1} a_{2} \ldots a_{n}$, $i \in\{1,2, \ldots, n-1\}: \sigma \in L \wedge a_{i}=a \wedge a_{i+1}=b$.

For example, from the logs given in Table 1, we have $A>_{L} B, A>_{L} C$, $C>_{L} D, B>_{L} D$, and so on.

\subsection{Log-based business process}

The sequence of activities in a $\log$ trace $\sigma=a_{1} a_{2} \ldots a_{n} \in A^{*}$ presents their ordering relations. A relation between an activity $a_{i}$ and its followed activity $a_{i+1}$ in the trace $\sigma, 1 \leq i \leq n-1$ can be presented as a directed edge from $a_{i}$ to $a_{i+1}$. The activity relations in a business process $\log L$ can be presented as a weighted directed graph where the edge's weight presents the number of times that the edge was repeated in the $\log L$. This graph is called log-based business process graph (Definition 4).

${ }^{4} \mathcal{P}\left(A^{*}\right)$ is the power set of $A^{*}$, i.e., $L \subseteq A^{*}$ 
Definition 4 (Log-based business process graph). A log business process graph is a weighted directed graph $G_{L}=\left(V_{L}, E_{L}, w\right)$ built from a business process $\log L^{*} \in \mathcal{P}^{*}\left(A^{*}\right)$ where:

- $V_{L}=A=\left\{a_{1}, a_{2}, \ldots, a_{n}\right\}$,

- $E_{L}=\left\{\left(a_{i}, a_{j}\right) \in A \times A: a_{i}>_{L} a_{j}\right\} \subseteq A \times A$,

- $w$ is a weight function from $E_{L}$ to $N$ :

$$
\begin{aligned}
w: E_{L} & \longrightarrow N \\
\left(a_{i}, a_{j}\right) & \mapsto\left|a_{i}>_{L} a_{j}\right|
\end{aligned}
$$

$\left|a_{i}>_{L} a_{j}\right|$ is number of times that $a_{i}>_{L} a_{j}$ comes about in the $\log L^{*}$

$w\left(a_{i}, a_{j}\right)=0$ if $\nexists \sigma=a_{1} a_{2} \ldots a_{n}, k \in\{1,2, \ldots, n-1\}: a_{k}=a_{i} \wedge a_{k+1}=a_{j}$

For example, the log-based business process graphs of the event logs given in Table 1 and Table 2 are depicted in Fig. 4 . The weight of each flow is the number of times that the flow is executed. It is emphasized by the arrow's thickness.

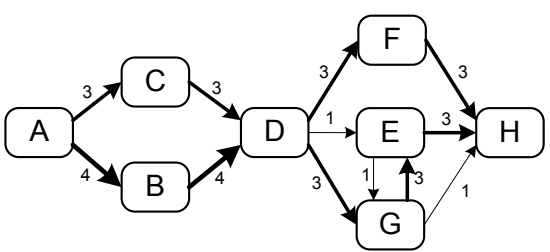

(a) the liability claim process

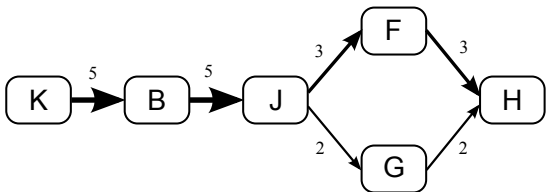

(b) the customer subscription process

Fig. 4: Log-based business process graphs

The log-based graph presents the execution of a business process in reality, regardless its conceptual model. The weights of edges present their execution frequency which indicates the strength of relations between activities. In the following, we present our approach to build the neighborhood context of an activity based on these relations.

\subsection{Log-based neighborhood context}

We define the log-based neighborhood context as a directed labeled graph that presents the shortest path from an activity to its neighbors. Intuitively, the closeness between activities is presented by the paths connecting them. The shortest path between activities presents their closest relation. The log-based neighborhood context of an activity presents the best relations between the activity and its neighbors.

In a log-based neighborhood context graph, each vertex is associated to a number that indicates the shortest path length from it to the associated activity. Vertexes that have the same shortest path length are considered to be located on the same layer around the associated activity. Thus, we name the number 
associated to each activity in a neighborhood context graph layer number. The layer number of an activity $a$ is denoted by $l(a)$. The area limited between two adjacent layers is called zone. The edge connecting two vertexes in a neighborhood context graph belongs to a zone as the vertexes are on the same or adjacent layers. We assign to each edge a number, so-call zone number, which determines the zone that the edge belongs to.

The edge connecting $a_{j}, a_{k}$ in the neighborhood context graph of an activity $a_{i}$ is assigned a zone number $z\left(a_{j}, a_{k}\right)=\min \left(l\left(a_{j}\right), l\left(a_{k}\right)\right)+1$. This means, if $a_{j}$ and $a_{k}$ are located on two adjacent layers, the edge $\left(a_{j}, a_{k}\right)$ will belongs to the zone limited by $l\left(a_{j}\right)$ and $l\left(a_{k}\right)$. In the case that $a_{j}$ and $a_{k}$ are located on the same layer, the edge connecting them belongs to the outer zone of their layer, which is limited by layers $l\left(a_{j}\right)$ and $l\left(a_{j}\right)+1$.

Definition 5 (Activity neighborhood context graph). The neighborhood context graph of an activity $a_{i}$, denoted by $G_{C}\left(a_{i}\right)$, is an extension of the log-based graph $G_{L}=\left(V_{L}, E_{L}, w\right)$ with vertex layer numbers and edge zone numbers. The layer number of an vertex $a_{j}$, denoted by $l\left(a_{j}\right)_{G_{C}\left(a_{i}\right)}$, is the shortest path length from $a_{j}$ to $a_{i}$ and the zone number of an edge $\left(a_{j}, a_{k}\right)$, denoted by $z\left(a_{j}, a_{k}\right)_{G_{C}\left(a_{i}\right)}$, has value $\min \left(l\left(a_{j}\right)_{G_{C}\left(a_{i}\right)}, l\left(a_{k}\right)_{G_{C}\left(a_{i}\right)}\right)+1$ :

1. $l\left(a_{j}\right)_{G_{C}\left(a_{i}\right)}=$ ShortestPathLength $\left(a_{j}, a_{i}\right)$,

2. $z\left(a_{j}, a_{k}\right)_{G_{C}\left(a_{i}\right)}=\min \left(l\left(a_{j}\right)_{G_{C}\left(a_{i}\right)}, l\left(a_{k}\right)_{G_{C}\left(a_{i}\right)}\right)+1, a_{j}>_{L} a_{k} \vee a_{k}>_{L} a_{j}$.

For example, the neighborhood context graphs of activity $D$ and $J$ in Fig. 4 are depicted in Fig. 5.

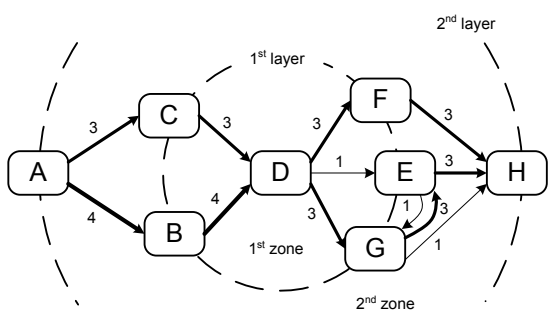

(a) the liability claim process

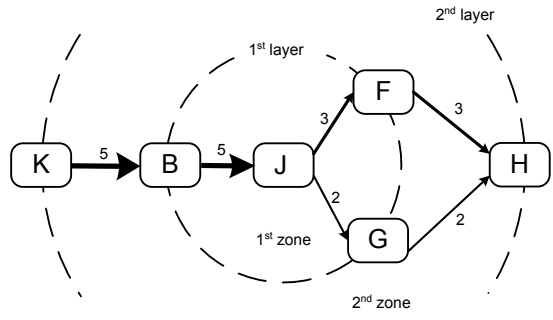

(b) the customer subscription process

Fig. 5: Neighborhood context graphs of the given event logs

Definition 6 ( $k^{\text {th }}$-neighbor). a is the $k^{\text {th }}$-neighbor of $b$, iff $l(a)_{G_{C}(b)}=k$. Set of $k^{t h}$-neighbors $(k \geq 1)$ of an activity $a_{i}$ is denoted by $N^{k}\left(a_{i}\right) . N^{k}\left(a_{i}\right)=\left\{a_{j}\right.$ : $\left.l\left(a_{j}\right)_{G_{C}\left(a_{i}\right)}=k\right\}$.

For example, in Fig. 4a, $N^{1}(A)=\{B, C\}, N^{2}(A)=\{D\}, N^{3}(A)=\{F, E, G\}$, $N^{1}(D)=\{C, B, F, E, G\}, N^{2}(D)=\{A, H\}, N^{3}(D)=\varnothing$, and so on.

\section{Activity recommendation based on neighborhood context matching}

The layer number and the zone number in a neighborhood context graph present the closeness between activities, while the weights of edges in the log-based graph 
present the strength of their relations. In this section, we detail our approach with log-based neighborhood context matching and activity recommendation.

\subsection{Neighborhood context matching}

To compute the matching between two neighborhood contexts, we (1) compute the matching of their edges in each zone, (2) multiply this matching with a zone-weight value and (3) sum up the matching in all zones.

We apply the vector space model (VSM) to compute the matching of edges in each zone of two neighborhood context graphs. VSM is a common technique used in Information Retrieval to compute the similarity between two items. It presents items in vectors and compute their similarity based on the cosine of the angle between the two corresponding vectors. In our approach, we present each zone as a vector of which elements are edges and values are their corresponding weights. Then, we align elements that connect the same activities in the same layers. Next, we present these vectors in the same space by filling 0 values in corresponding positions of the unaligned elements. Finally, we compute the cosine value of these two zone-vectors.

Particularly, in the first zone, we match the edges that connect the two associated activities to the same activities in the first layer. To formalize our computation, we define the two associated activities as root activities and name them $r_{0}$.

Concretely, assume that $P_{p}$ and $P_{q}$ are two log-based business processes constructed from event $\operatorname{logs} L_{p}$ and $L_{q}$. Let $A_{p}, A_{q}$ be sets of activities of $P_{p}$ and $P_{q}$ respectively. We compute the similarity between activities $a \in A_{p}$ and $b \in A_{q}$ by applying VSM as following.

Let $E_{P_{p}}^{k}(a)$ and $E_{P_{q}}^{k}(b)$ be sets of edges in $k^{\text {th }}$-zone of $a \in P_{p}$ and $b \in P_{q}$ respectively. Let $\overrightarrow{e(a)}, \overrightarrow{e(b)}$ be corresponding zone vectors.

$$
\begin{aligned}
E_{P_{p}}^{k}(a) & =\left\{(x, y): z(x, y)=k, x, y \in A_{p}\right\} \\
& =\left\{\left(x_{1}, y_{1}\right),\left(x_{2}, y_{2}\right), \ldots,\left(x_{m}, y_{m}\right)\right\} \\
\overrightarrow{e(a)} & =\left(w\left(x_{1}, y_{1}\right), w\left(x_{2}, y_{2}\right), \ldots, w\left(x_{m}, y_{m}\right)\right) \\
E_{P_{q}}^{k}(b) & =\left\{(e, f): z(e, f)=k, e, f \in A_{q}\right\} \\
& =\left\{\left(e_{1}, f_{1}\right),\left(e_{2}, f_{2}\right), \ldots,\left(e_{n}, f_{n}\right)\right\} \\
\overrightarrow{e(b)} & =\left(w\left(e_{1}, f_{1}\right), w\left(e_{2}, f_{2}\right), \ldots, w\left(e_{n}, f_{n}\right)\right)
\end{aligned}
$$

Let $N_{c}^{k-1}(a, b)$ and $N_{c}^{k}(a, b)$ be the sets of common neighbors of $a$ and $b$ on layers $k-1$ and $k, k>0$. We have:

$$
\begin{aligned}
N_{c}^{k-1}(a, b) & =N_{P_{p}}^{k-1}(a) \cap N_{P_{q}}^{k-1}(b) \\
N_{c}^{k}(a, b) & =N_{P_{p}}^{k}(a) \cap N_{P_{q}}^{k}(b)
\end{aligned}
$$

As we define the two associated activities as root activities and name them $r$, we have: $N_{P_{p}}^{0}(a)=a=r_{0}, N_{P_{q}}^{0}(b)=b=r_{0}$ and $N_{c}^{0}(a, b)=r_{0}$. 
Let $E_{c}^{k}$ be the set of common edges of $a$ and $b$ in $k^{t h}$-zone.

$$
\begin{aligned}
E_{c}^{k}= & \left\{(r, t):\left(r \in N_{c}^{k-1}(a, b), t \in N_{c}^{k}(a, b) \mid r>_{L_{p}} t \wedge r>_{L_{q}} t\right)\right. \\
& \left.\cup\left(r \in N_{c}^{k}(a, b), t \in N_{c}^{k-1}(a, b) \mid r>_{L_{p}} t \wedge r>_{L_{q}} t\right)\right\} \\
= & \left\{\left(r_{1}, t_{1}\right),\left(r_{2}, t_{2}\right), \ldots,\left(r_{m}, t_{m}\right)\right\}
\end{aligned}
$$

Let $\overrightarrow{e_{c}(a)}, \overrightarrow{e_{c}(b)}$ be vectors of weights of these common edges.

$$
\begin{aligned}
& \overrightarrow{e_{c}(a)}=\left(w\left(r_{1}, t_{1}\right), w\left(r_{2}, t_{2}\right), \ldots, w\left(r_{z}, t_{z}\right)\right),\left(r_{i}, t_{i}\right) \in E_{L}\left(A_{p}\right), 1 \leq i \leq z \\
& \overrightarrow{e_{c}(b)}=\left(w\left(r_{1}, t_{1}\right), w\left(r_{2}, t_{2}\right), \ldots, w\left(r_{z}, t_{z}\right)\right),\left(r_{i}, t_{i}\right) \in E_{L}\left(A_{q}\right), 1 \leq i \leq z
\end{aligned}
$$

By applying VSM, the similarity between $a$ and $b$ in the $k^{t h}$ zone is given by Equation. 1.

$$
M^{k}(a, b)=\frac{\overrightarrow{e_{c}(a)} \cdot \overrightarrow{e_{c}(b)}}{|\overrightarrow{e(a)}| \times|\overrightarrow{e(b)}|}
$$

For example, we have: the common neighbors of $D$ and $J$ in the $1^{\text {st }}$-layer are $N_{c}^{1}(D, J)=\{F, G, B\}$. So, $\overrightarrow{e_{c}(D)}=(w(D, F), w(D, G), w(B, D))=(3,3,4)$, $\overrightarrow{e_{c}(J)}=(w(J, F), w(J, G), w(B, J))=(3,2,5)$ and their matching in the $1^{s t}$-zone is:

$$
M^{1}(D, J)=\frac{3 \times 3+3 \times 2+4 \times 5}{\sqrt{3^{2}+1^{2}+3^{2}+3^{2}+4^{2}} \times \sqrt{3^{2}+2^{2}+5^{2}}}=0.86
$$

In the $2^{n d}$-zone, we have the common edges of these two context graphs are: $(F, G)$ and $(G, H)$. So, their matching in this zone is:

$$
M^{2}(D, J)=\frac{3 \times 3+1 \times 2}{\sqrt{3^{2}+4^{2}+3^{2}+3^{2}+1^{2}+1^{2}+3^{2}} \times \sqrt{5^{2}+3^{2}+2^{2}}}=0.24
$$

The behavior of an activity is strongly reflected by the connections to its closet neighbors. Therefore, we propose to consider a zone weight in our matching. Concretely, as the zone-weight has to have greater values in smaller $k^{\text {th }}$ connection zone, we propose to assign the zone-weight a value computed by a polynomial function which is $w_{j}^{z}=\frac{k+1-j}{k}$, where $j$ is the zone number $(1 \leq j \leq k), k$ is the number of considered zones around an associated activity. The closest zone to the associated activity has a weight 1 and the farthest zone has a weight $\frac{1}{k}$. The final matching formula improved with the zone weight consideration is given in Equation 2.

$$
M^{*}(a, b)=\frac{2}{k+1} \times \sum_{i=1}^{k} \frac{k+1-i}{k} \times M^{i}(a, b)
$$

For example, the matching between the neighborhood contexts of $D$ and $J$ (in 2 zones) with zone weights is:

$$
M^{*}(D, J)=\frac{2}{3} \times\left(M^{1}(D, J)+\frac{1}{2} \times M^{2}(D, J)\right)=\frac{2}{3} \times\left(0.86+\frac{1}{2} \times 0.24\right)=0.65
$$




\subsection{Activity recommendation}

The neighborhood context graph presents the interactions between the associated activity and its neighbors in layers. It can infer the behavior of the associated activity. Therefore, the matching between neighborhood context graphs exposes the similarity between the associated activities. In our approach, the higher the matching value is, the more similar the activities are. Basically, the steps to make recommendations based on log-based neighborhood context matching are:

1. We represent the business execution logs in a log-based graph. This graph contains relations between activities and their weight values.

2. For each activity in the log-based graph, we build a neighborhood context graph which contains the closet relations between the associated activity and its neighbors. In a neighborhood context graph, activities are presented in layers and relations between them are presented in zones.

3. We compute the matching between neighborhood context graphs using vector space model. This matching presents the similar between two corresponding activities in terms of relations to their neighbors.

4. Finally, for a selected activity, we sort other activities in descending order of similarity and pick up top- $n$ activities for recommendation.

\subsection{Computational complexity}

In our approach, only the connection flows connecting common neighbors in two adjacent layers are taken into account for the matching computation. So, by using queues (data structure) to store the common neighbors and track them from the nearest layers to the furthest layers, we avoid the redundant checking of unrelated neighbors. On the other hand, the number of activities as well as the number of common neighbors in a log-based business process are not great ${ }^{5}$, our algorithm can run fast in computing the neighborhood context matching of two activities. The worst case of this algorithm's computation time is $\mathcal{O}\left(n_{A} \times n_{P} \times n \times k\right)$, where $n_{A}$ is the number of activities, $n_{P}$ is the number of business processes, $n$ is the maximum number of common activities located on a layer and $k$ is the number of considered layers. The worst case only happens when all the business processes in the system are entirely matched. In addition, the performance of the algorithm can be improved by processing the neighborhood context matching periodically off-line.

\section{Implementation and experiments}

To validate our approach, we implement a tool that generates recommendations based on business process logs. We also perform experiments on a large public dataset shared by the IBM Business Integration Technologies (BIT) team. Details of our implementation (section 5.1) and experiments (section 5.2) are presented as follows.

${ }^{5}$ On making statistics on the public dataset used in our experiment, we have that in average, there are 11.36 services in a business process (section 5) 


\subsection{Implementation}

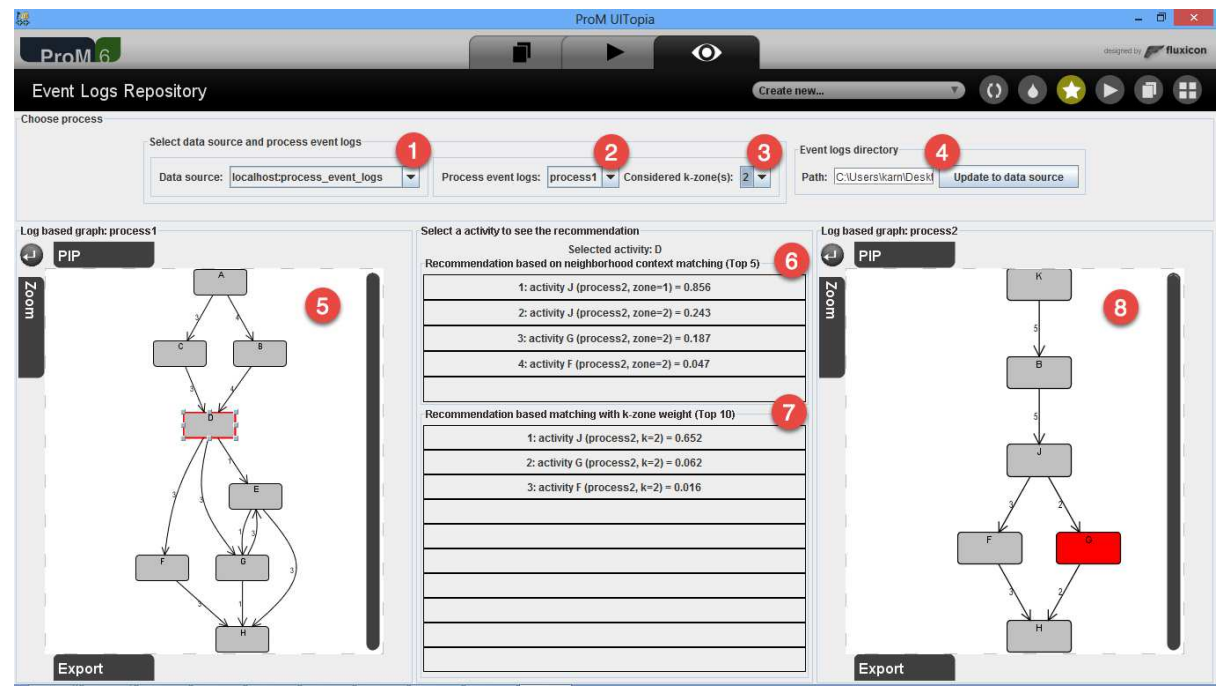

Fig. 6: A screen shot of the log-based activity recommendation application

We implemented an activity recommendation plug-in and integrated it into ProM. This plug-in interacts with either a database or log files to retrieve recommendations for each selected activity in the ProM interface. This application ${ }^{6}$ was developed to validate our approach as a proof of concept.

The screen-shot of our application is shown in Fig. 6. It includes of 8 areas. Areas 1 and 4 allow selecting event log resources, which can be either from a database or a log files directory. The designer selects a working process in area 2 and specifies a zone number to be considered by the algorithm in area 3 . The working process is shown in area 5 . The designer can select an activity in the working process to obtain recommendations. Area 6 shows the top- 5 recommended activities without zone-weight in the computation. Meanwhile, area 7 shows the top-10 recommended activities with zone-weight consideration. If the designer selects a recommended activity in area 6 or 7 , this activity and its involved process will be shown in area 8 (the selected activity is highlighted).

\subsection{Experiments}

A big challenge of our approach is the availability of real business process logs. We attempted to search and contact other research groups for both public and private logs. However, process event logs are not published or they are not under a disclosure agreement. There are very few logs that are shared for the competition of the BPI challenge ${ }^{7}$. But they are not usable in our approach as they are just logs of one business process while we need logs of several different processes.

\footnotetext{
${ }^{6}$ published at: http://www-inf.it-sudparis.eu/SIMBAD/tools/LogRec/
}

7 http://www.win.tue.nl/bpi/ 
We performed experiments on logs generated from real business processes. Data of these processes were shared by the IBM Business Integration Technologies (BIT) team. This dataset includes 735 different processes in Petri net Markup Language (PNML) format. These processes are designed for insurance, banking, customer relationship, as well as construction and automotive supply chain domains [20].

We transformed the collected business process models from PNML format to the Colored Petri Net (CPN) format. Then, we used CPN Tools ${ }^{8}$ to load the transformed process models to generate process event logs, which are resulted in XES files. Finally, these log files were imported by our application, which is a ProM plugin, to display the log-based business process graphs, compute the similarity between activities and show recommendations (as presented in Fig. 6). Details of the log-based dataset is given in Table 3.

\begin{tabular}{|l|l|l|l|}
\hline & Min. & Max. & Average \\
\hline Number of process instances of a process model & 50 & 299 & 189.88 \\
\hline Number of occurring events in a process & 31 & 8283 & 1004.15 \\
\hline Number of events in a process & 2 & 64 & 9.02 \\
\hline Number of start events in a process & 1 & 5 & 1.11 \\
\hline Number of end events in a process & 1 & 13 & 2.56 \\
\hline
\end{tabular}

Table 3: Details of the dataset in XES format

We perform two experiments to evaluate the feasibility and the accuracy of our approach. In the first experiment, we vary the $k^{t h}$-zone values from 1 to 5 and make statistics on the number of recommended activities for each selected activities. Fig. 7 shows the percentage of activities that have at least 1 recommended activity with the similarity value is greater than $0,0.5$ and 0.8 .

Concretely, we obtained that more than $76.56 \%$ activities that have at least one similarity value greater than 0 with $k$ varies from 1 to 5 . With $k=1$, we obtained $53.68 \%$ activities with similarity value greater than 0.5 and $23.31 \%$ activities with similarity value greater than 0.8 . These results show that our approach can provide recommendations for a majority activities as we can retrieve

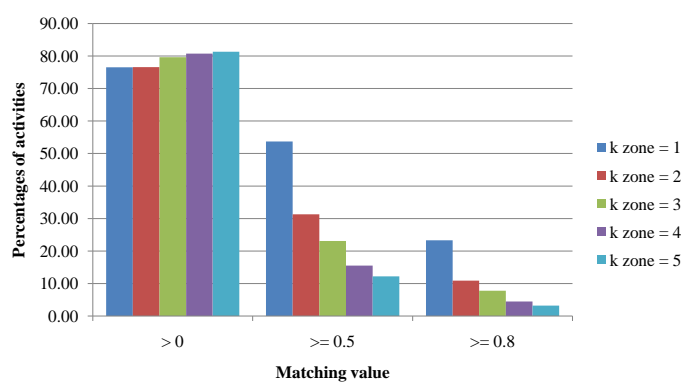

Fig. 7: Percentage of activities that have at least one recommended activity with the similarity value greater than $0,0.5$ and 0.8 .

\footnotetext{
${ }^{8} \mathrm{CPN}$ Tools is an application to generate synthetic process event logs from models described in CPN format. It is available at: http://cpntools.org/
} 
similar activities for more than $3 / 4$ number of activities in average. It means that our approach is feasible and can be applied in real use-cases.

In the second experiment, we evaluate the accuracy of our approach based on Precision and Recall metrics. As our approach takes into account neighborhood contexts instead of activity identifiers, we consider activity identifiers as groundtruth data in computing Precision and Recall. Concretely, consider a selected activity $a$ in a log-based business process $P$. Assume that $a$ appears in $n \log$ based business processes. The recommendations for this selected position consist of $l$ activities, in which $t(t \leq l)$ activities are $a$. Precision and Recall of these recommendations are given by Equation 3.

$$
\text { Precision }=\frac{t}{l} ; \quad \text { Recall }=\frac{t}{n}
$$

The primary objective of the experiment is to retrieve a small share of activities that are likely irrelevant (high precision). It is of secondary importance to retrieve the full range of potentially relevant activities (moderate recall) in order to avoid the designer being overwhelmed.

In our experiment, we computed the Precision and Recall with $l$ (number of activities recommended for each selected activity) equal to 1, 4, 7 and 10 . We performed the experiment with $k^{t h}$-zone $=1$ and on activities that appear in at least $2,5,7$ and 10 different business processes.
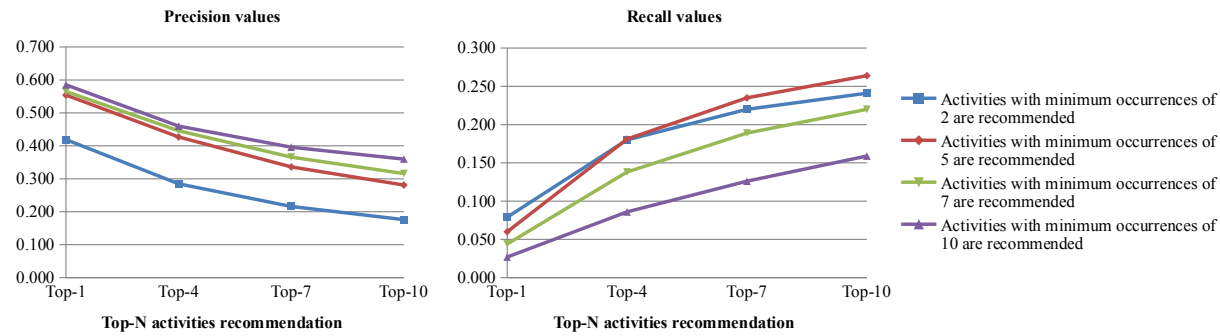

Fig. 8: Precision and Recall values of activities that are occurrence in at least 2, 5,7 and 10 different processes, $k^{\text {th }}$-zone $=1$ and $l$ is equal to $1,4,7$ and 10

Fig. 8 shows that we obtained good Precision values (from 0.42 to 0.59 ) in case of recommending 1 activity for each selected activity. These values decrease when we increase the number of recommended activities. On the other hand, activities that appear in more processes will have greater Precision values. It also shows that the Recall

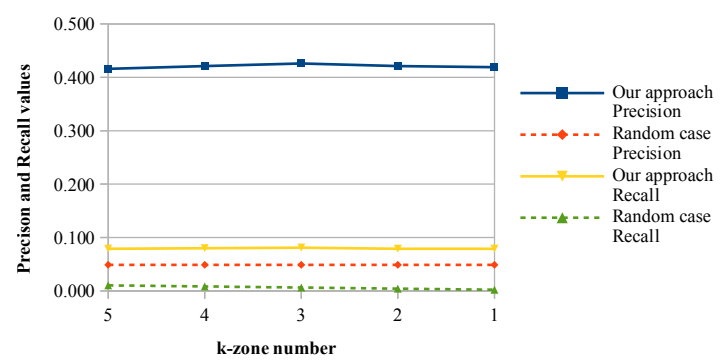

Fig. 9: Average Precision and Recall values with different zone layers 
values increase when $l$ (or top- $\mathrm{N}$ ) changes from 1 to 10 . This means that we can retrieve more relevant activities when the number of recommended activities increases. The highest Recall value in our experiment is 0.264 when $l=10$.

Fig. 9 shows the average Precision and Recall values of our approach with different $k^{\text {th }}$-zone values. It shows that our approach achieved much better results than an approach that generates recommendations randomly (in average, 8.6 times greater the Precision value and 17.4 times greater the Recall value).

The Precision and Recall values showed that our approach retrieve not only the right activities but also new relevant activities for each selected position and the number of new activities increases when the number of recommended activities increases. These results showed that our approach can be applied in real use-cases as we can provide the designer the flexibility in using existing process fragments or designing new process variants.

\section{Related work}

Computing the similarity of two process models first requires the definition of a mapping between the activities using automatic matching techniques [17]. Principles for matching process models have been integrated in the ICoP framework [33] and extended with semantic matching techniques [23]. Applications in the area of supporting process model design adopt such semantic techniques, as for instance the approach based on the process descriptor space [25] and the recommendation approach reported in [21]. Approaches to harmonize structure [27] and labels [24] were also proposed. Service recommendation $[9,10]$ can be also applied in the context of activity recommendation. Behavioral abstractions such as trace adjacency $[5,36]$ and weak order relations $[19,34]$ provide a means to compare process behavior $[15,22,16]$. These notions are applied, e.g., for identifying connections between actions [30] comparable to our notion of activity neighborhood. Our approach builds on this observation to make recommendations for executable processes. In addition, we focus on process fragments instead of the entire model. We recommend activities instead of process models and we do not face the computational complexity problem.

In our previous work [11-13], we exploited activity relations in process models and we did not take into account the occurrence frequency of activities. In this work, we realize that knowledge from process logs can be also utilized for process design by applying process mining techniques. Different techniques have been defined for automatically discovering whole models from logs, e.g. [1,26, 18]. The automatic matching between event logs and process models is discussed in [6] showing that logs on the execution level are often much more detailed than models. The challenge of process mining is the observation that process models often turn out to be overwhelmingly complex, so-called spaghetti models [2]. The approach reported in this paper helps to present correlations between activities in a context-specific way, which allows us to hide the complexity of the behavior. Hidden knowledge in process event logs are discovered for assisting business process design. In this way, we complement log-based recommendation approaches to support process designers at runtime [7] or at configuration time [8]. 


\section{Conclusion}

In this paper, we addressed the challenge of supporting the designer during the act of modeling, even in cases where no comparable process models exist. We present an approach that effectively utilizes knowledge extracted from business process logs for recommending activities. This approach is based on a notion of activity neighborhood and a corresponding calculation of similarity. The approach has been implemented as a plug-in for ProM and evaluated using generated log data of 735 processes from practice.

In future work, we aim to extend the similarity calculation with other properties of an activity such as descriptions, actors, resources and dependencies. We also plan to integrate our neighborhood matching technique with a specific query language to help to retrieve activities that have similar contexts based on a requested activity context.

\section{References}

1. van der Aalst, W., Weijters, T., Maruster, L.: Workflow mining: Discovering process models from event logs. Knowl. and Data Eng. (9), 1128-1142 (Sep 2004)

2. van der Aalst, W.M.P.: Process Mining - Discovery, Conformance and Enhancement of Business Processes. Springer (2011)

3. Aalst, W., Lohmann, N., La Rosa, M., Xu, J.: Correctness ensuring process configuration: an approach based on partner synthesis. In: BPM. pp. 95-111 (2010)

4. Aalst, W., Medeiros, A., Weijters, A.: Process equivalence: Comparing two process models based on observed behavior. In: BPM. pp. 129-144 (2006)

5. Bae, J., Liu, L., Caverlee, J., Zhang, L.J., Bae, H.: Development of distance measures for process mining, discovery and integration. Int. J. Web Service Res. 4(4), $1-17(2007)$

6. Baier, T., Mendling, J.: Bridging abstraction layers in process mining by automated matching of events and activities. In: BPM (2013)

7. Barba, I., Weber, B., Valle, C.D., Ramirez, A.J.: User recommendations for the optimized execution of business processes. Data Knowl. Eng. 86, 61-84 (2013)

8. Buijs, J.C.A.M., van Dongen, B.F., Aalst, W.M.P.: Mining configurable process models from collections of event logs. In: BPM '13 (2013)

9. Chan, N.N., Gaaloul, W., Tata, S.: Web services recommendation based on user's behavior. In: ICEBE. pp. $214-221$ (nov 2010)

10. Chan, N.N., Gaaloul, W., Tata, S.: A web service recommender system using vector space model and latent semantic indexing. In: AINA. pp. $602-609$ (2011)

11. Chan, N.N., Gaaloul, W., Tata, S.: Composition context matching for web service recommendation. In: SCC. pp. 624-631 (2011)

12. Chan, N.N., Gaaloul, W., Tata, S.: Context-based service recommendation for assisting business process design. In: EC-Web '11, pp. 39-51 (2011)

13. Chan, N.N., Gaaloul, W., Tata, S.: Assisting business process design by activity neighborhood context matching. In: ICSOC (2012)

14. Curran, T., Keller, G., Ladd, A.: SAP R/3 business blueprint: understanding the business process reference model. Upper Saddle River, NJ, USA (1998)

15. Dijkman, R., Dumas, M., Garcia-Banuelos, L.: Graph matching algorithms for business process model similarity search. In: BPM. pp. 48-63 (2009) 
16. Dijkman, R.M., van Dongen, B.F., Dumas, M., García-Bañuelos, L., Kunze, M., Leopold, H., Mendling, J., Uba, R., Weidlich, M., Weske, M., Yan, Z.: A short survey on process model similarity. In: Seminal Contributions to Information Systems Engineering. Springer (2013)

17. Ehrig, M., Koschmider, A., Oberweis, A.: Measuring similarity between semantic business process models. In: Conceptual Modelling 2007, APCCM2007. CRPIT, vol. 67, pp. 71-80. Australian Computer Society (2007)

18. Engel, R., van der Aalst, W., Zapletal, M., Pichler, C., Werthner, H.: Mining interorganizational business process models from edi messages: A case study from the automotive sector. Advanced Inf. Sys. Engineering (2012)

19. Eshuis, R., Grefen, P.W.P.J.: Structural matching of bpel processes. In: ECOWS 2007. pp. 171-180. IEEE Computer Society (2007)

20. Fahland, D., Favre, C., Jobstmann, B., Koehler, J., Lohmann, N., Völzer, H., Wolf, K.: Instantaneous soundness checking of industrial business process models. In: 7th BPM. pp. 278-293 (2009)

21. Hornung, T., Koschmider, A., Lausen, G.: Recommendation based process modeling support: Method and user experience. In: ER '08. pp. 265-278 (2008)

22. Kunze, M., Weske, M.: Metric trees for efficient similarity search in large process model repositories. In: zur Muehlen, M., Su, J. (eds.) BPM Workshops. Lecture Notes in Business Information Processing, vol. 66. Springer (2010)

23. Leopold, H., Niepert, M., Weidlich, M., Mendling, J., Dijkman, R., Stuckenschmidt, H.: Probabilistic optimization of semantic process model matching. In: BPM (2012)

24. Leopold, H., Smirnov, S., Mendling, J.: On the refactoring of activity labels in business process models. Inf. Syst. 37(5), 443-459 (2012)

25. Lincoln, M., Golani, M., Gal, A.: Machine-assisted design of business process models using descriptor space analysis. In: BPM. pp. 128-144 (2010)

26. Maggi, F., Bose, R., Aalst, W.: Efficient discovery of understandable declarative process models from event logs. In: Advanced Information Systems Engineering, vol. 7328, pp. 270-285. Springer Berlin Heidelberg (2012)

27. Polyvyanyy, A., García-Bañuelos, L., Dumas, M.: Structuring acyclic process models. Inf. Syst. 37(6), 518-538 (2012)

28. Rozinat, A., Mans, R.S., Song, M., van der Aalst, W.M.P.: Discovering colored petri nets from event logs. Int. J. Softw. Tools Technol. Transf. (1), 57-74 (2007)

29. Schonenberg, H., Weber, B., Dongen, B., Aalst, W.: Supporting flexible processes through recommendations based on history. In: BPM. pp. 51-66 (2008)

30. Smirnov, S., Weidlich, M., Mendling, J., Weske, M.: Action patterns in business process model repositories. Computers in Industry (2012)

31. Stephens, S.: Supply chain operations reference model version 5.0: A new tool to improve supply chain efficiency and achieve best practice. Information Systems Frontiers pp. 471-476 (December 2001)

32. Van Der Aalst, W.M.P.: Configurable services in the cloud: supporting variability while enabling cross-organizational process mining. In: OTM. pp. 8-25 (2010)

33. Weidlich, M., Dijkman, R., Mendling, J.: The icop framework: identification of correspondences between process models. In: 22nd CAiSE. pp. 483-498 (2010)

34. Weidlich, M., Mendling, J., Weske, M.: Efficient consistency measurement based on behavioral profiles of process models. IEEE Trans. Soft. Eng. 37, 410-429 (2011)

35. Yan, Z., Dijkman, R., Grefen, P.: Fast business process similarity search with feature-based similarity estimation. In: OTM. pp. 60-77 (2010)

36. Zha, H., Wang, J., Wen, L., Wang, C., Sun, J.: A workflow net similarity measure based on transition adjacency relations. Computers in Industry 61(5), 463-471 (2010) 